\section{Das Weiterbenutzungsrecht an verlängerten Patenten ${ }^{1}$.}

\section{Von Patentanwalt Dr. B. Alexander-Katz, Berlin-Görlitz.} (Bingeg. 29,;3. 1922.)

Zweck des Verlängerungsgesetzes vom 27. April 1920 ist, den Frfinder oder den Schutzrechtinhaber durch Verlängerung der gesetzlichen Schutzdauer für die Nachteile zu entschädigen, die ihm bei der Verwertung seines Schutzrechtes durch die Kriegsverhältnisse erwachsen sind. Das durch die Verlängerung erneute Ausschlußrecht wird durch $\S 7$ des Gesetzes im Sinne des Vorbenutzerrechtes des Patentgesetzes zugunsten derjenigen beschränkt, welche mit dem gesetzlichen Ablauf der Schutzdauer gerechnet, sich darauf eingerichtet und die durch Erlosschen des Schutzrechtes frei gewordene Erfindung in Benutzung genommen hatten. Diese Benutzer sollten durch die nicht vorauszusehende Verlängerung des Schutzrechtes einem Eingriff des Erfinders in ihre wohlerworbenen Rechte nicht ausgesetzt sein. Die Frage, wie weit der Kreis dieser Begünstigten zu ziehen ist, ist heftig umstritten. Das Verlängerungsgesetz macht die freie Weiterbenutzung des verlängerten Schutzrechtes davon abhängig, daß es vor dem 1. April 1920 und nach seinem Erlöschen in Benutzung genommen, oder wenigstens die zur Benutzung erforderliche Veranstaltung vor dieser Zeit getroffen sein muß. In der Literatur ist vielfach erörtert und verschieden beurteilt worden, ob auch demjenigen das Weiterbenutzungsrecht $z u$ gewähren ist, der, sei es infolge eines Lizenzvertrages oder im Wege der Verletzung, das Schutzrecht vor seinem Erlöschen benutzt und die Benutzung nach Erlöschen, aber vor dem 1. April 1920 fortgesetzt hat. Diese Frage ist nach $Z$ weck und Inhalt des Verlängerungsgesetzes zu verneinen. Wollte man auch dem Lizenznehmer und dem Verletzer ein kostenloses Weiterbenutzungsrecht gewähren, so würde der Kreis der Begünstigten so weit gezogen, daß der oben angegebene Zweck des Verlängerungsgesetzes vereitelt würde. Es liegt im Interesse des Erfinders, daß der Kreis der Begünstigten so eng wie möglich gezogen wird, wenn er eine wirksame Entschädigung für die ihm durch die Kriegsverhältnisse entstandene Schmälerung der Nutzung aus seinem Schutzrecht erhalten soll.

Weder der Lizenznehmer noch der Verletzer erfullen die Voraussetzungen, an welche das Gesetz die kostenlose Weiterbenutzung knüpft. Das Schutzrecht muß nach $\& 7$ des Verlängerungsgesetzes erst durch Erlöschen frei und jedermann dadurch zugänglich geworden sein, bevor die Benutzung begonnen hat. Wer bereits vor Ablauf des Schutzrechtes die Erfindung widerrechtlich benutzt und den Erfinder dadurch um seinen Lohn gebracht hat, ist in den Kreis der aus $\$ 7$ Berechtigten nicht einbezogen, obwohl er die Benutzung nach Ablauf des Schutzrechtes und vor dem kritischen Zeitpunkt, dem 1. April 1920, fortgesetzt hat. Maßgebend ist, daß er mit der Benutzung selbst nicht gewartet hat, bis er hierzu nach Erjöschen des Schutzrechtes berechtigt war. Nur die Vorbereitung der Benutzung darf auch vor dem Zeitpunkt des Erlöвchens gemäß $§ 7$, Absatz 2 des Gesetzes liegen, nicht aber der Beginn der Benutzung. Es wäre unbillig, wenn man dem Verletzer das Weiterbenutzungsrecht einräumen und demjenigen, der sich auf die Vorbereitung der Benutzung beschränkt und mit der Benutzung selbst bis zum Erloschen des Schutzrechtes gewartet hat, das Weiterbenutzungrecht nur gegen angemessene Vergütung gewähren wollte. Dem Verletzer steht nicht einmal der Anspruch auf Benutzung gegen angemessene Vergütung $\mathrm{zu}$, da er tiber die bloße Vorbereitung vor Ablauf des Schutzrechtes hinausgegangen ist.

Der entgegenstehenden Auffassung des O. L. G. Celle kann nicht beigetreten werden. Das Urteil vom 16. Januar 1920 erkennt wohl an, da $B$ das Verlängerungsgesetz den Eingriff des Inhabers des erneuerten Schutzrechtes in Rechte dritter Benutzer verhindern soll, soweit diese Rechte durch Benutzung ehemals geschutzter, längst frei gewordener Erfindungen wohl erworben sind. Hiertaber besteht kein Streit. In dem Fall, der dem Urteil zugrunde liegt, handelt es sich aber nicht um die Benutzung, die erst nach Freiwerden des Schutzrechtes einsetzt, sondern um die unberechtigte Benutzung einer gesch int $\Delta$ ten Erfindung und die Fortsetzung derselben nach Erlöschen des Schutzrechtes. Es liegt danach in diesem Falle ein wohlerworbenes Recht im Sinne des $\& 7$ des Verlängerungsgesetzes nicht vor.

Ebenso wie der Verletzer scheidet auch der Lizenznehmer aus dem Kreise der Begunstigten aus. Auch auf ihn treffen die Voraus-

1) Zur Erläuterung dieses Aufsatzes bemerkt der palentkundige Berater der Schriftleitung folgendes: „Bekanntlich war gemäB Gesetz vom 27. April 1920 dem Inhaber eines Patents oder eines Gebrauchsmusters Gelegenheit gegeben, seine Schutzrechte um die Kriegsdauer zu verlängern. Er mußte innerhalb einer bestimmten Frist einen begründeten Antrag stellen, über den ein besonderer, im Patentamt gebildeter AusschuB beschloB. Die Verlängerung geschah in der Weise, daß die Zeit vom 1. August 1914 bis 3F. Juli 1919 nicht auf die Dauer des Schutzrechtes angerechnet wird.

Der in diesem Aufsatz besprochene $\$ 7^{\circ}$ dieses Gesetzes regelt die Rechte des Vorbenutzers, ähnlich wie dies der $\$ 5$ des Patentgesetzes für die Patente allgemein tut. Nach dem $\$ 7$ ist derjenige, der vor dem 1. April 1920 die Erfindung, nachdem das Scbutzrecht erloschen war, im Inland in Benutzung genommen, oder wer vor diesem T'ag im Inland die zar Benulzung erforderlichen Veranstaltungen getroffen hat, auch nach der Verlängerung berechtigt, die Erfindung für die Bedürfnisse seines eigenen Betriebes weiter zu benutzen.

Näheres hierzu ,Zeitschr. f. angew. Chemie 1920, Aufsatzteil, S. 145". setzungen, welche das Gesetz an die kostenfreie Weiterbenutzung knupft, nicht zu. Er hat gleichfalls mit der Benutzung der Erfindung nicht erst nach Erlöschen des Schutzrechtes begonnen, sondern die Erfindung - wenn auch berechtigt - vorher benutzt und nachher fortgesetzt. Seine Rechte werden durch eine besondere Bestimmung gemäß \& 8 des Verlängerungsgesetzes geregelt. Sein Lizenzrecht war spätestens mit dem Ablauf der normalen Dauer des Patentes erloschen, und er hat nur Anspruch auf Erneuerung dieses Rechtes unter bestimmten Voraussetzunzen. Der Gesetzgeber wollte ihn also bewußt vom freien Weiterbenutzungsrecht ausschließen. Dieser besonderen Regelung hätte es nicht erst bedurft, wenn der Lizenznehmer auch ohne Erneverung des Lizenzvertrages schon aus $\$ 7$ zur kostenfreien Weiterbenutzung bereshtigt wäre. Allerdings erscheint es hart, daß der Lizenzrehmer ungünstiger gestellt sein soll, als der Dritte, der die Erfindung frei weiterbenutzen darf. Er hat aber vor Ablauf des Schutzrechtes mehr oder weniger lange die Möglichkeit gehabt, unter dem Schutz des Patentes Vorteile aus der Benutzung zu ziehen, die von ihm gemachten Aufwendungen auszunutzen und sich einen Kundenkreis urd Absatz zu sichern, den der andere gutgläubige freie Weiterbenulzer sich erst unter erheblichen Aufwendungen, die er im guten Glauben an da; Freiwerden des Schutzrechtes gemacht hat, erwerben muß. Der Lizenz.nehmer genic St den Patentschutz, wenn auch durch die freien Weiterbenutzer beschränkt, nach wie vor und ist daher verpflichtet, für die weitere Benutzung des erneuerten Schutzes dem Schutzinhaber eine Vergütung zu gewähren. Danach zählen wede $r$ der Verletzer, noch der Lizenznehmer zu den aus $\$ 7$ des Verlängt rungsgesetzes berechtigten kostenfreien Weiterbenutzern.

[A. 88.]

\section{Die Extraktion geringer Flüssigkeitsmengen im Soxhletschen Extraktionsapparat.}

\author{
(D.R.G.M. 807757 und 809512.)
}

Von Dr. Heinrich Handorf, Hamburg.

(Eingeg. 31./3. 1822.)

Zur permanenten Extraktion geringer Flüssigkeitsmengen kamen bisher Sonderkonstruktionen des Soxhletschen oder ăhnlicher Extraktionsapparate in Frage, teils erheblicher Kompliziertheit, die als solche für diesen $Z$ weck beschafft werden midssen, und nur für diesen Zweck Verwendung finden können, wăhrend sich der - wohl uberall vorhandene - Originalapparat zu diesem $\mathrm{Z}$ weck nur heranziehen läßt, wenn man die zu extrahierende Flussigkeit z. B. von gebranntem Gips aufnehmen läßt, um alsdann die durchfeuchtete und dann getrocknete Substanz in den gebräuchlichen Extraktionshülsen auf übliche Weise auszuziehen; mit welchem Erfolge, ist bekannt ${ }^{1}$ ).

Bei den hier wiedergegebenen Vorrichtungen handelt es sich um zwei Einsătze, welche für den Originalapparat nach Soxhlet sowie für seine sinngemäBen Modifikationen, soweit sie zur Extraktion fester Stoffe gedacht sind, passen und diese Geräte zur Extraktion kleiner Flussigkeitsmengen mit spezifisch leichteren oder schwereren Lossungsmitteln dienstbar machen wollen. DaB das Lösungsmittel mit der auszuziehenden Flüssigkeit nicht wesentlich mischbar ist, bleibt dabei Voraussetzung.

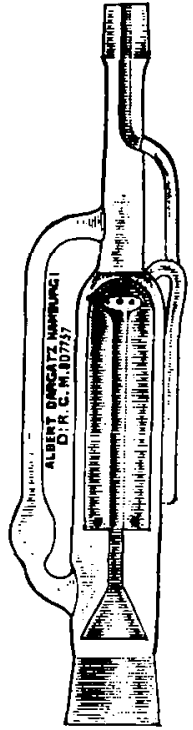

Fig. 1.

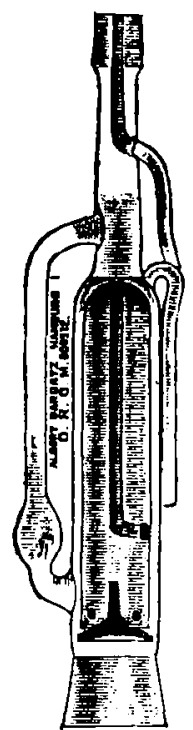

Fig. 2.
Der in Abb. 1 wiedergegebene Apparat arbeitet nur mit Lösungsmitteln, welche leichter sind als die zu extrahierende Flussigkeit (Äthermodell). Seine Wirkungsweise ist folgende: Bei Inbetriebnahme des Einsatzes wird das Becherglas mit der zu extrahierenden

1) Vgl. Gadamer, Lehrhuch der ehemischen Toxikologie, 1909, S. 363. 